\title{
$I-V$ curves of $\mathrm{Fe} / \mathrm{MgO}(001)$ single- and double-barrier tunnel junctions
}

\author{
J. Peralta-Ramos, ${ }^{1, *}$ A. M. Llois, ${ }^{1,2}$ I. Rungger, ${ }^{3}$ and S. Sanvito ${ }^{3}$ \\ ${ }^{1}$ Departamento de Física, Centro Atómico Constituyentes, Comisión Nacional de Energía Atómica, Buenos Aires, Argentina \\ ${ }^{2}$ Departamento de Física, Facultad de Ciencias Exactas y Naturales, Universidad de Buenos Aires, Buenos Aires, Argentina \\ ${ }^{3}$ School of Physics and CRANN, Trinity College, Dublin 2, Ireland
}

(Received 30 March 2008; revised manuscript received 1 July 2008; published 23 July 2008)

\begin{abstract}
In this work, we calculate with $a b$ initio methods the current-voltage characteristics for ideal single- and double-barrier Fe/MgO (001) magnetic tunnel junctions. The current is calculated in the phase-coherent limit by using the recently developed SMEAGOL code, combining the nonequilibrium Green function formalism with density-functional theory. In general we find that double-barrier junctions display a larger magnetoresistance, which decays with bias at a slower pace than their single-barrier counterparts. This is explained in terms of enhanced spin filtering from the middle Fe layer sandwiched in between the two MgO barriers. In addition, for double-barrier tunnel junctions, we find a well defined peak in the magnetoresistance at a voltage of $V$ $=0.1 \mathrm{~V}$. This is the signature of resonant tunneling across a majority quantum well state. Our findings are discussed in relation to recent experiments.
\end{abstract}

DOI: 10.1103/PhysRevB.78.024430

PACS number(s): 85.75.- d, 72.25.Mk, 73.40.Rw, 73.23.Ad

\section{INTRODUCTION}

Tunneling magnetoresistance (TMR) arises in magnetic tunnel junctions consisting of an insulating barrier sandwiched by two ferromagnetic electrodes. It is observed that the resistance strongly depends on the relative orientation of the magnetization vectors of the electrodes. It is usually small when the magnetizations are parallel to each other and increases when they are antiparallel, and the change in resistance can be large. The TMR coefficient is defined as $\mathrm{TMR}=\left[\left(I_{\mathrm{P}}-I_{\mathrm{AP}}\right) / I_{\mathrm{AP}}\right] \times 100$, where $I_{\mathrm{P}}$ and $I_{\mathrm{AP}}$ are the currents in the parallel $(\mathrm{P})$ and in the antiparallel (AP) magnetic configurations related to the same applied voltage, respectively. Since the pioneering work of M. Jullière ${ }^{1}$ and Fabian et al. ${ }^{2}$ the experimentally attainable TMR values have been steadily increasing mainly due to the tremendous advances in growth techniques. In particular a steep increase in TMR magnitude followed from the growth of highly crystalline $\mathrm{Fe} / \mathrm{MgO}$ (001) tunnel junctions with atomically flat surfaces. ${ }^{3}$ Currently the record value is $500 \%$ at room temperature in fully epitaxial $\mathrm{FeCoB} / \mathrm{MgO} / \mathrm{FeCoB}$ junctions. ${ }^{4}$ This value is much larger than the one that can be obtained in standard metallic giant magnetoresistive devices. This explains why magnetic tunnel junctions will soon replace metallic spin valves in the read heads of near-future hard disk drives. $^{5}$

In epitaxial single-barrier magnetic tunnel junctions (SBMTJs), the large TMR is a consequence of the symmetry matching between Bloch states in the electrodes and the complex bands within the insulator. ${ }^{6}$ The latter are evanescent states lying in the band gap and decaying inside the spacer. For thin spacers, another transport mechanism may arise when surface states at each side of the barrier come into resonance. These form the so-called hot spots in the transmission coefficient as a function of the transverse wave vector $k_{\|}$. This mechanism was clearly demonstrated in the $a b$ initio calculations of MacLaren et al. ${ }^{7}$ for Fe/MgO SBMTJs, in which a $\mathrm{Fe}$ minority surface state was found resonant through the $\mathrm{MgO}$ spacer. It was then shown experimentally by Tiusan $e t a l .{ }^{8}$ and theoretically by Rungger $e t a l .{ }^{9}$ that, in $\mathrm{Fe} / \mathrm{MgO}$ SBMTJs, these surface states are very sensitive to the applied bias voltage $V$, and, as a consequence, the TMR is maximal at zero bias and significantly decreases with increasing bias. The bias voltage at which the TMR drops to half of its zero-bias value is denoted as $V_{1 / 2}$ and it is taken as one of the quality factors for the applicability of SBMTJs in real devices. Typically $V_{1 / 2}$ is less than $0.7 \mathrm{~V}$.

Nowadays, one of the main challenges in the field of spin electronics is to reach simultaneously large TMR and $V_{1 / 2}$ values. One possible route to accomplish this goal was proposed by Zhang et al..$^{10}$ a decade ago and consists of the insertion of a metallic slab in between the insulating spacer, thus forming a double-barrier magnetic tunnel junction (DBMTJ). In this architecture, quantum well states (QWSs) formed by confinement are found in the in-between metallic slab. These can come into resonance with the evanescent states inside the barriers, enhancing the TMR in a significant way and allowing the tuning of the TMR by controlling the thickness of the in-between slab. This tunability of the TMR arises because changing the thickness of the in-between metallic slab produces a shift in the energy of the QWS and creates new ones. In addition, since the potential drop is shared across the two barriers and an additional spindependent scattering potential is introduced by the inbetween magnetic slab, the $V_{1 / 2}$ for DBMTJs is expected to be larger than that of a SBMTJ of same combined thickness.

To date there have been several theoretical studies on the spin-dependent properties of DBMTJs (Refs. 11-14) with either magnetic or nonmagnetic in-between slabs. Usually these are conducted at zero bias in the linear-response limit or in a non-self-consistent fashion. The key features emerging from these studies are: first that the TMR can reach extremely large values under resonant conditions and second that the TMR can be enhanced not only by resonant tunneling through QWS but also by the spin-filter effect (SFE). ${ }^{14}$ This effect is a consequence of the insertion of a magnetic slab in between the barriers. In fact in these DBMTJs, the P configuration corresponds to all the magnetizations (the two 
electrodes and the in-between layer) being aligned parallel to each other while in the AP configuration, the electrode magnetizations remain parallel to each other but the in-between magnetization is antiparallel to them. Thus in the AP configuration of DBMTJs, there are effectively two interfaces where the magnetization vector change sign and this greatly enhances backscattering. Since the electrodes and the inbetween Fe layers have different coercive fields due to their different thicknesses, these magnetic configurations $\mathrm{P}$ and AP are experimentally attainable. ${ }^{5}$

The SFE has been clearly shown in our previous calculations for ideal Fe/ZnSe (001) SBMTJs and DBMTJs with Fe in-between layers. ${ }^{14}$ The main difference between resonant tunneling through QWS and the SFE is that the former strongly depends on the thickness of the in-between slab while the latter is almost independent. Thus, DBMTJs show a variety of spin-dependent transport phenomena richer than that of their single-barrier counterparts and, in principle, they promise a better control of the TMR. Some of these expectations are now confirmed experimentally. For example, Nozaki et al. ${ }^{15}$ have recently measured the $I-V$ curves of fully epitaxial $\mathrm{Fe} / \mathrm{MgO}$ SBMTJs and DBMTJs (with Fe as the in-between metallic slab), and found that (i) the TMR of DBMTJs is larger than that of SBMTJs and (ii) the TMR decrease with bias is significantly slower in DBMTJs than in SBMTJs. Similarly Zeng et al. ${ }^{16}$ have shown that $\mathrm{CoFeB} /$ Al-O DBMTJs also have TMR and $V_{1 / 2}$ values larger than those of the corresponding SBMTJs. Other works along the same lines that confirm these results include that of Ref. 17.

In spite of the amount of research carried out on the spindependent transport properties of DBMTJs, self-consistent calculations of $I-V$ curves for a realistic junction from first principles are still lacking. The purpose of this paper is to fill this gap. We present $a b$ initio calculated $I-V$ characteristics for $\mathrm{Fe} / \mathrm{MgO}$ (001) DBMTJs and compare them to those of SBMTJs, relating the transport properties of the devices to their electronic structure. For this goal, we use the recently developed $a b$ initio code SMEAGOL, ${ }^{18}$ which combines the pseudopotential density-functional code SIESTA (Ref. 19) for the electronic structure calculations with the nonequilibrium Green function formalism (NEGF) for phase-coherent transport. ${ }^{20}$

\section{CALCUlation DETAILS}

Our SBTJs consist of $n$ monolayers (MLs) of MgO (001) sandwiched by two semi-infinite bcc Fe (001) electrodes while our DBMTJs are multilayers of the type $(\mathrm{MgO})_{n} / \mathrm{Fe}_{m} /(\mathrm{MgO})_{n}(001)$, sandwiched by the same electrodes. In both cases, the junctions are assumed to be periodic in the $x-y$ plane with $z$ being the transport direction. In order to account for the charge transfer, and to correctly reproduce the band offset between $\mathrm{Fe}$ and $\mathrm{MgO}$, we include in the cell, for self-consistent calculations, four Fe MLs belonging to the electrodes at both sides of the junction. This is enough to correctly account for charge screening inside the ferromagnet. Similar to previous calculations, ${ }^{7,9,13}$ the lattice constant of the electrodes is fixed to $2.87 \AA$ and that of $\mathrm{MgO}$ is taken to be $\sqrt{2}$ larger. This, together with a $45^{\circ}$ rotation of
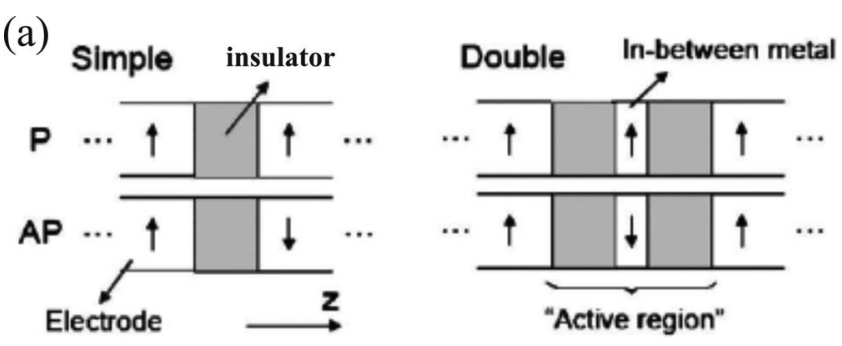

(b)
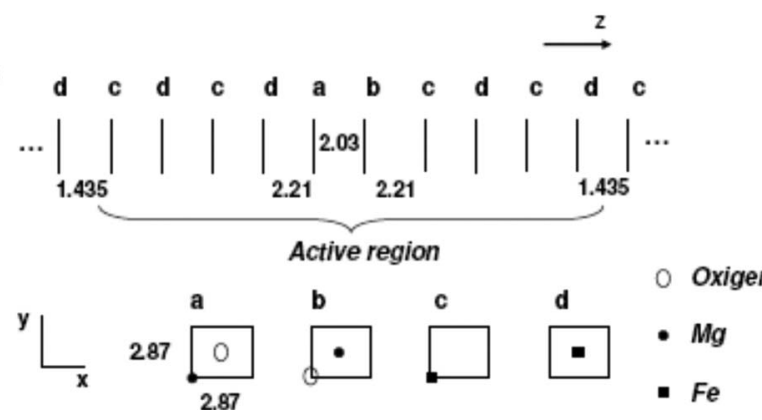

FIG. 1. (a) Structure of single- and double-barrier junctions. The arrows indicate the two magnetic configurations considered (parallel and antiparallel). (b) Structure of a SBMTJ with $n=2$ ML, showing the active region and part of the semi-infinite $\mathrm{Fe}$ electrodes. The junction is periodic in the $x-y$ plane while $z$ is the transport direction. Distances are in angstrom.

the Fe unit cell, allows epitaxial matching between $\mathrm{Fe}$ and $\mathrm{MgO}$. Figure 1 shows the structure of a single-barrier junction with $n=2$ ML (4 $\AA$ ) together with the schematic structure of single- and double-barrier junctions. In this work, the possible appearance of $\mathrm{FeO}$ interfacial layers, as well as atomic relaxation and disorder, are not considered so that our calculations are valid only in the ballistic limit for atomically ordered structures.

For the electronic structure of the junctions, we use normconserving pseudopotentials, double-zeta basis set for all the angular momenta, and generalized gradient approximation (GGA) (Ref. 21) on the exchange and correlation potentials. We have thoroughly checked that the band structure and the density of states of bulk $\mathrm{Fe}$, bulk $\mathrm{MgO}$, and $\mathrm{Fe} / \mathrm{MgO}$ multilayers, as well as the charge transfer and magnetic moments in the last case, are very well reproduced as compared to full potential linear augmented plane wave (FP-LAPW) results obtained using the highly accurate WIEN2K code. ${ }^{22}$ We obtain a band offset (the difference between the Fermi energy $E_{F}$ of $\mathrm{Fe}$ and the valence band of $\mathrm{MgO}$ ) of $3.51 \mathrm{eV}$, in very good agreement with previous theoretical ${ }^{23}$ and experimental reports. ${ }^{24}$ It is well-known that density-functional calculations using semilocal exchange and correlation functionals underestimate the band gap, and ours are not an exception. We obtain a band gap of $5.4 \mathrm{eV}$ [as compared to the experimental value of $7.8 \mathrm{eV}$ (Refs. 24 and 25)], which agrees well with what was expected from GGA. ${ }^{7,23}$

The ballistic current density at each bias voltage $V$ is calculated as

$$
I^{\sigma}(V)=\frac{e}{h} \int d E T^{\sigma}(E, V)\left[f_{L}-f_{R}\right],
$$

where $f_{L}=f\left(E-\mu_{L}\right) \quad\left[f_{R}=f\left(E-\mu_{R}\right)\right]$ is the Fermi-Dirac function evaluated at $E-\mu_{L}\left(E-\mu_{R}\right)$ with $\mu_{L}=E_{F}+e V / 2$ 


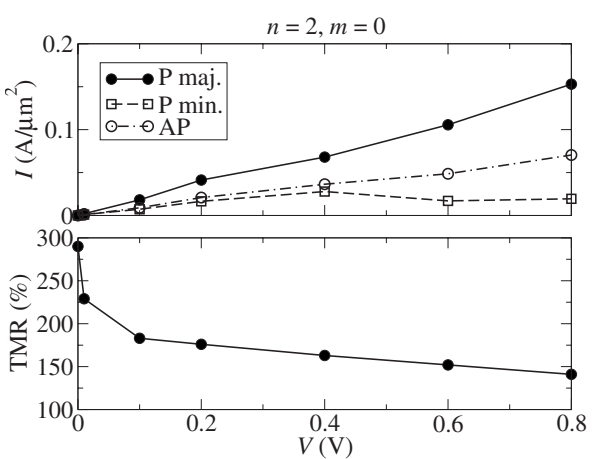

FIG. 2. Current density and TMR as a function of bias voltage of the single-barrier junction with $n=2$ ML.

$\left(\mu_{R}=E_{F}-e V / 2\right)$ as the chemical potential of the left (right) electrode. Finally $\sigma$ is the spin index standing for majority $(\sigma=\uparrow)$ and minority $(\sigma=\downarrow)$ spins. The transmission coefficient $T^{\sigma}(E, V)$ is calculated for each bias and it is given by

$$
T^{\sigma}(E, V)=\frac{1}{V_{B Z}} \int d k_{x} d k_{y} T^{\sigma}\left(E, V, k_{x}, k_{y}\right),
$$

where $V_{\mathrm{BZ}}$ is the area of the two-dimensional (2D) Brillouinzone orthogonal to the transport direction. Here we assume that both spin and transverse momentums are conserved, an approximation that is valid for relatively thin epitaxial junctions. The $k_{\|}$-resolved transmission coefficient appearing in Eq. (2) $\left(\vec{k}_{\|}=k_{x} \hat{x}+k_{y} \hat{y}\right.$, see Fig. 1) is calculated from the nonequilibrium Green function formalism in the usual way. ${ }^{18,20}$ It is given by $\operatorname{T}=\operatorname{Tr}\left(\Gamma_{L} G^{r} \Gamma_{R} G^{a}\right)$, where for simplicity we omit the spin label $\sigma$. Here, $\Gamma_{L, R}$ are the broadening matrices describing the interaction (thus the finite lifetime) of the scattering region energy levels with the left- and right-hand side electrodes, and $G^{r}\left(G^{a}\right)$ is the associated retarded (advanced) Green function describing the one-electron electronic structure of the scattering region. The broadening matrices are calculated from the self-energies $\Sigma_{L, R}$ as $\Gamma_{L, R}=i\left(\Sigma_{L, R}-\Sigma_{L, R}^{\dagger}\right)$. These in turn are obtained with the semianalytic method described in Refs. 26 and 27.

In our calculations, we use a $8 \times 8 \times 8 k$-point mesh in reciprocal space to calculate the density matrix of the scattering region and a $150 \times 150 \times 1$ mesh to evaluate the current at each bias voltage. We have carefully verified that these meshes are sufficient for converging the density matrix and the current.

\section{RESULTS}

\section{A. Single-barrier tunnel junctions}

In order to benchmark our calculations for DBMTJs, we have calculated first the conductance and TMR at zero, and finite bias of several single-barrier $\mathrm{Fe} / \mathrm{MgO}$ (001) junctions as a function of the barrier thickness $n$. In general we find a rapid decrease in the TMR as a function of bias in agreement with previous theoretical results. ${ }^{9}$

As an example, Fig. 2 shows the current-voltage curve for $n=2$ ML (4 A) together with the corresponding TMR. At zero bias, the TMR is calculated from the transmission prob-

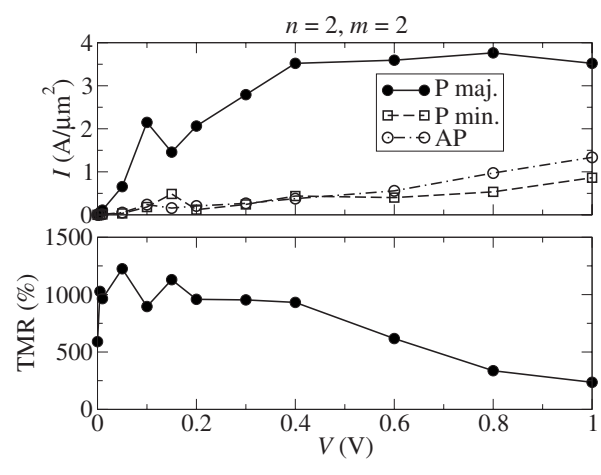

FIG. 3. Current density and TMR as a function of bias voltage for a double-barrier magnetic tunnel junction with $n=2 \mathrm{ML}$ and $m=2$ ML.

abilities [Eq. (2)] evaluated at $E_{F}$. It is seen that the currents for each spin channel are almost linear functions of $V$ over the bias range investigated. However, the $\mathrm{P}$ minority current saturates at $V=0.4 \mathrm{~V}$ while the $\mathrm{P}$ majority and the AP currents keep increasing beyond that value. The competition between the rates of increase as a function of bias of the $\mathrm{P}$ majority and AP currents produces a rapid decay of the TMR, and we calculate $V_{1 / 2}$ being at around $0.7 \mathrm{~V}$. The TMR peaks at zero bias where it reaches up to $290 \%$ but, because of its almost exponential decay, it approaches the mean value over the bias range investigated of $160 \%$ within only $0.2 \mathrm{~V}$. All of these features are in good agreement with experimental data ${ }^{15,28,29}$ and early theoretical results. ${ }^{30,31}$ It is important to note also that, at very low bias voltages, the $\mathrm{P}$ minority current is slightly larger than the AP current. As it was shown by Rungger et al., ${ }^{9}$ this large P minority current near the zero bias is due to the resonance of a $\mathrm{Fe}$ minority surface state through the $\mathrm{MgO}$ barrier and it is washed out as soon as a voltage is applied across the device.

\section{B. Double-barrier tunnel junctions}

Figures 3 and 4 show the current density and the TMR for junctions with $n=2 \mathrm{ML}$, and $m=2 \mathrm{ML}(2.87 \AA)$ and $m$ $=4 \mathrm{ML}(5.74 \AA)$, respectively. The first thing to notice is the appearance of oscillations in the TMR at low bias. These are not present in the SBMTJ and stem from peaks in the low

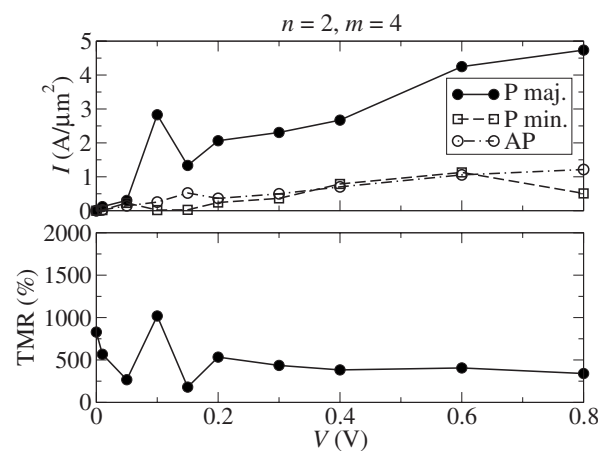

FIG. 4. Current density and TMR as a function of bias voltage for a double-barrier magnetic tunnel junction with $n=2 \mathrm{ML}$ and $m=4$ ML. 


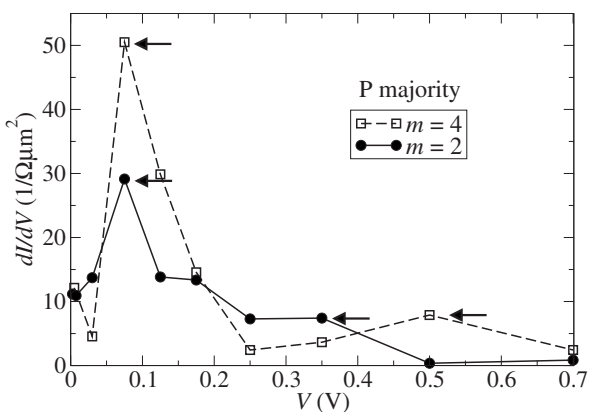

FIG. 5. Differential conductance associated to the majority current in the $P$ configuration for DBMTJs with $m=2$ MLs and $m=4 \mathrm{ML}$ as a function of bias.

bias $I$ - $V$ curve for all the spin channels. It is seen that the $\mathrm{P}$ majority current has a large peak at $V=0.1 \mathrm{~V}$, present for both values of $m$. Although the $\mathrm{P}$ minority and the AP currents also present peaks as a function of bias, these are considerably smaller than those in the $\mathrm{P}$ majority channel. A difference between the two junctions investigated is that the bias at which the peaks appear depends on the number of monolayers $m$ of the in-between Fe layer. Thus, the TMR oscillations as a function of bias are different in the two cases. In particular, for $m=2$, there are two TMR peaks at $V=0.05 \mathrm{~V}$ and at $V=0.15 \mathrm{~V}$, at which the TMR reaches values close to $1250 \%$. At $V=0.1 \mathrm{~V}$, the $\mathrm{P}$ majority current peak competes with that of the AP configuration, resulting in the actual decrease in the TMR. In contrast, for $m=4$, there is a very large TMR peak at $V=0.1 \mathrm{~V}$, which clearly originates from the corresponding one in the P majority current.

The peak in the P majority current of DBMTJs is suggestive of resonant tunneling through a QWS positioned in the in-between Fe slab. This is consistent with the experimental reports of Nozaki et al. ${ }^{32}$ for Fe/MgO (001) DBMTJs with Fe islands as the in-between slab. They observed small conductance oscillations with bias only in the P configuration. In particular, for the thinnest in-between Fe slab investigated (1 $\mathrm{nm})$, they observed two peaks in the $\mathrm{P}$ differential conductance at $V \sim 0.15$ and $\sim 0.4 \mathrm{~V}$. Very recently, Wang et al. ${ }^{13}$ have theoretically shown that these conductance oscillations could be fitted to majority QWSs of $\Delta_{1}$ symmetry at the $\Gamma$ point once the level shift produced by charging effects is taken into account. As it can be seen in Fig. 2(a) of Ref. 13 for ultrathin in-between Fe slabs, there is a majority QWS at the $\Gamma$ point with an energy slightly less than $E_{F}+0.1 \mathrm{eV}$. It is then tempting to ascribe our P majority peak at $V=0.1 \mathrm{~V}$ to resonant tunneling through the above mentioned QWS.

Although our intention in this work is not to push for a quantitative agreement with the results of Nozaki et al. ${ }^{32}$ it is interesting to compute the differential conductance of our DBMTJs for the P majority channel. These are shown in Fig. 5. The differential conductance is defined as $G=d I / d V$ and in this work it is simply calculated numerically from the $I-V$ curves. From Fig. 5 it is seen that both DBMTJs show a conductance peak around $V=0.1 \mathrm{~V}$. There is also a second conductance peak occurring at around $V=0.35 \mathrm{~V}$ for $m$ $=2 \mathrm{ML}$ and at around $V=0.5 \mathrm{~V}$ for $m=4 \mathrm{ML}$. Importantly the bias voltages at which these peaks occur are rather close to the experimental ones even if our DBMTJs have significantly thinner in-between Fe slabs.

A second intriguing feature of the DBMTJs is that the $\mathrm{P}$ majority current does not increase linearly with bias, in contrast to what happens for both the $\mathrm{P}$ minority and AP currents. As a consequence the TMR decay is not as severe as in the case of single-barrier junctions. In addition to that, it is important to report that the AP current for DBMTJs is significantly reduced with respect to that of the SBMTJs. For the simple SBMTJ investigated here, this is always larger than the P minority current except at very low bias where the resonance of $\mathrm{Fe}$ minority surface state plays the dominant role. ${ }^{9}$ In contrast, for double-barrier junctions, the AP and the $\mathrm{P}$ minority currents are almost equal to each other even at relatively high voltages. This feature increases the bias voltage at which the TMR starts to decay, pushing $V_{1 / 2}$ to higher biases. That is to say, our DBMTJs can sustain large TMR values even under the application of high bias voltages. As it was mentioned in the introduction, the reduction in the AP current is an indication that the SFE originated from the insertion of a magnetic slab. We then conclude that the spinfilter effect plays an important role in the TMR decay with bias in double-barrier junctions.

By comparing the DBMTJs with the SBMTJ, it is clear that the TMR of DBMTJs does not only decay slower but it is also considerably higher than that of the SBMTJ with the same barrier thickness. This is due to the reduction in AP current in DBMTJs, which originated from the spin-filter effect. ${ }^{14}$ Therefore, the SFE in DBMTJs increases both $V_{1 / 2}$ and the TMR. These two results, together with the TMR oscillations at low bias mentioned above, are the most important features of our DBMTJs. It would be interesting to study the $I-V$ curves of DBMTJs with a very thick inbetween Fe slab since, according to our picture based on the SFE, the TMR decay with bias would be significantly reduced and the TMR values would increase.

\section{CONCLUSIONS}

In summary, we have compared first-principles calculations of the $I-V$ characteristics of single and double $\mathrm{Fe} / \mathrm{MgO}$ (001) magnetic tunnel junctions in the phase-coherent spinconserving transport regime. Our results are in semiquantitative agreement with recent experiments and demonstrate that: (i) the TMR for double-barrier junctions is significantly higher than that of single-barrier not only at low but also for finite bias, (ii) the TMR decay with bias voltage is slower in double-barrier than in single-barrier junctions, and (iii) double-barrier junctions exhibit low bias TMR oscillations. We have shown that the spin-filter effect is important in understanding the origin of the features (i) and (ii) while the third aspect is related to resonant electron tunneling through a majority-spin QWS formed in the in-between Fe slab. In our opinion the first two aspects are the most relevant for real DBMTJs since they are rather robust with respect to changes 
in the junction geometry. Thus, a possible route to obtain a very large TMR, which does not depend strongly on the bias voltage, is that of fabricating double-barrier junctions with very thick in-between magnetic slabs. This is a potentially useful strategy for constructing spin-transfer element where one needs to increase the current density and preserve a high degree of spin polarization.

\section{ACKNOWLEDGMENTS}

This work was partially funded by UBACyT-X115, PIPCONICET 6016, PICT 05-33304, and PME 06-117. I.R. and S.S. give thanks to Science Foundation of Ireland for financial support (Grant No. SFI 07/IN.1/I945). A.M.L. belongs to CONICET (Argentina).
*Corresponding author.peralta@cnea.gov.ar

${ }^{1}$ M. Jullière, Phys. Lett. 54, 225 (1975).

${ }^{2}$ J. Fabian, A. Matos-Abiague, C. Ertler, P. Stano, and I. Zutić, Acta Phys. Slov. 57, 565 (2007); arXiv:0711.1461 (unpublished).

${ }^{3}$ S. Yuasa and D. D. Djayaprawira, J. Phys. D 40, R337 (2007).

${ }^{4}$ Y. M. Lee, J. Hayakawa, S. Ikeda, F. Matsukura, and H. Ohno, Appl. Phys. Lett. 90, 212507 (2007).

${ }^{5}$ G. D. Fuchs, N. C. Emley, I. N. Krivorotov, P. M. Braganca, E. M. Ryan, S. I. Kiselev, J. C. Sankey, D. C. Ralph, R. A. Burman, and J. A. Katine, Appl. Phys. Lett. 85, 1205 (2004).

${ }^{6}$ P. H. Mavropoulos, N. Papanikolaou, and P. H. Dederichs, Phys. Rev. Lett. 85, 1088 (2000).

${ }^{7}$ J. M. MacLaren, X.-G. Zhang, W. H. Butler, and X. Wang, Phys. Rev. B 59, 5470 (1999); W. H. Butler, X.-G. Zhang, T. C. Schulthess, and J. M. MacLaren, ibid. 63, 054416 (2001).

${ }^{8}$ C. Tiusan, J. Faure-Vincent, C. Bellouard, M. Hehn, E. Jouguelet, and A. Schuhl, Phys. Rev. Lett. 93, 106602 (2004).

${ }^{9}$ I. Rungger, A. R. Rocha, O. Mryasov, O. Heinonen, and S. Sanvito, J. Magn. Magn. Mater. 316, 481 (2007).

${ }^{10}$ X. Zhang, B.-Z. Li, G. Sun, and F.-C. Pu, Phys. Rev. B 56, 5484 (1997).

${ }^{11}$ A. G. Petukhov, A. N. Chantis, and D. O. Demchenko, Phys. Rev. Lett. 89, 107205 (2002); N. Ryzhanova, G. Reiss, F. Kanjouri, and A. Vedyayev, Phys. Lett. A 329, 392 (2004).

${ }^{12}$ M. Wilczyński, J. Barnaś, and R. Świrkowicz, J. Magn. Magn. Mater. 267, 391 (2003); W.-S. Zhang, B.-Z. Li, and Y. Li, Phys. Rev. B 58, 14959 (1998).

${ }^{13}$ Y. Wang, Z.-Y. Lu, X.-G. Zhang, and X. F. Han, Phys. Rev. Lett. 97, 087210 (2006).

${ }^{14}$ J. Peralta-Ramos and A. M. Llois, Phys. Rev. B 73, 214422 (2006).

${ }^{15}$ T. Nozaki, A. Hirohata, N. Tezuka, S. Sugimoto, and K. Inomata, Appl. Phys. Lett. 86, 082501 (2005).

${ }^{16}$ Z.-M. Zeng, X.-F. Han, W.-S. Zhan, Y. Wang, Z. Zhang, and S. Zhang, Phys. Rev. B 72, 054419 (2005).

${ }^{17}$ F. Montaigne, J. Nassar, A. Vaurès, F. Nguyen Van Dau, F. Petroff, A. Schuhl, and A. Fert, Appl. Phys. Lett. 73, 2829 (1998); S. Ohya, P. N. Hai, and M. Tanaka, ibid. 87, 012105 (2005); A. Iovan, S. Andersson, Yu. G. Naidyuk, A. Vedyaev, B. Dieny, and V. Korenivski, Nano Lett. 8, 805 (2008); J. H. Lee,
K.-I. Jun, K.-H. Shin, S. Y. Park, J. K. Hong, K. Rhie, and B. C. Lee, J. Magn. Magn. Mater. 286, 138 (2005).

${ }^{18}$ A. R. Rocha, V. M. García-Suárez, S. Bailey, C. J. Lambert, J. Ferrer, and S. Sanvito, Phys. Rev. B 73, 085414 (2006); A. R. Rocha, V. M. García-Suárez, S. Bailey, C. J. Lambert, J. Ferrer, and S. Sanvito, Nat. Mater. 4, 335 (2005).

${ }^{19}$ J. M. Soler, E. Artacho, J. D. Gale, A. García, J. Junquera, P. Ordejón, and D. Sánchez-Portal, J. Phys.: Condens. Matter 14, 2745 (2002).

${ }^{20}$ S. Datta, Electronic Transport in Mesoscopic Systems (Cambridge University Press, Cambridge, 1999); H. Haug and A.-P. Jauho, Quantum Kinetics in Transport and Optics of Semiconductors (Springer-Verlag, Berlin, 1996); C. Caroli, R. Combescot, P. Nozieres, and D. Saint-James, J. Phys. C 4, 916 (1971).

${ }^{21}$ J. P. Perdew, K. Burke, and M. Ernzerhof, Phys. Rev. Lett. 77, 3865 (1996).

${ }^{22}$ P. Blaha, K. Schwarz, G. Madsen, D. Kvasnicka, and J. Luitz, WIEN2k: An Augmented Plane Wave Plus Local Orbitals Program For Calculating Crystal Properties (Technical Universitat Wien, Wien, 2002).

${ }^{23}$ B. D. Yu and J.-S. Kim, Phys. Rev. B 73, 125408 (2006); K. D. Belashchenko, J. Velev, and E. Y. Tsymbal, ibid. 72, 140404(R) (2005).

${ }^{24}$ M. Klaua, D. Ullmann, J. Barthel, W. Wulfhekel, J. Kirschner, R. Urban, T. L. Monchesky, A. Enders, J. F. Cochran, and B. Heinrich, Phys. Rev. B 64, 134411 (2001).

${ }^{25}$ R. C. Whited, C. J. Flaten, and W. C. Walker, Solid State Commun. 13, 1903 (1973).

${ }^{26}$ S. Sanvito, C. J. Lambert, J. H. Jefferson, and A. M. Bratkovsky, Phys. Rev. B 59, 11936 (1999).

${ }^{27}$ I. Rungger and S. Sanvito, J. Magn. Magn. Mater. 316, 481 (2007).

${ }^{28}$ S. Yuasa, T. Nagahama, A. Fukushima, Y. Suzuki, and K. Ando, Nat. Mater. 3, 868 (2004).

${ }^{29}$ C. Tiusan, M. Sicot, M. Hehn, C. Belouard, S. Andrieu, F. Montaigne, and A. Schuhl, Appl. Phys. Lett. 88, 062512 (2006).

${ }^{30}$ J. Mathon and A. Umerski, Phys. Rev. B 63, 220403(R) (2001).

${ }^{31}$ D. Waldron, V. Timoshevskii, Y. Hu, K. Xia, and H. Guo, Phys. Rev. Lett. 97, 226802 (2006).

${ }^{32}$ T. Nozaki, N. Tezuka, and K. Inomata, Phys. Rev. Lett. 96, 027208 (2006). 